\title{
O perfil dos ingressantes de um programa de educação física para idosos e os motivos da adesão inicial
}

CDD. 20.ed. 613.7

796.018
João Raimundo Peixoto PEREIRA* Silene Sumire OKUMA*
* Escola de Educação Física e Esporte, Universidade de São Paulo.

\section{Resumo}

Numa sociedade em pleno envelhecimento, a Educação Física deverá desempenhar um importante papel. A cada ano, cresce a procura por programas supervisionados, como é o caso do Programa Autonomia para Atividade Física do Idoso - PAAF. Conhecer o idoso é fundamental para orientar as ações pedagógicas e atender suas expectativas. Assim, este trabalho destina-se a conhecer o perfil sócio-demográfico, a atividade física pregressa, verificar a saúde percebida e entender quais foram os motivos de adesão ao programa. Participaram desse estudo 54 pessoas ( 14 homens e 40 mulheres), com idade acima de 60 anos (66,45 $\pm 5,176$ anos de idade), que ingressaram no PAAF-2002. Foram utilizados questionários, em que foram consideradas as seguintes variáveis: gênero, idade, nivel de escolaridade, estado civil; saúde percebida do ingressante; a atividade profissional e a atividade física pregressa e o nível sócioeconômico. Para verificar os motivos da adesão inicial utilizamos tabela tipo Liekert e aplicamos o Índice de Consistência Interna de Liekert. Utilizamos o Coeficiente de Concordância de Kendall (W) e o Teste de Friedman $\left(X^{2}\right)$, com nivel de significância $(p=0,05)$. A maioria dos alunos tinha entre 60 a 70 anos de idade, pertencia aos níveis sócio-econômico A e B e possuía alto nível de escolaridade. A maioria tinha pelo menos uma doença, porém, a saúde percebida era positiva. Os motivos de adesão relatados foram: aprender fazer exercícios físicos, melhorar condicionamento físico e prevenir problemas de saúde. Já, ocupar o tempo livre e fazer amigos foram motivos considerados menos importantes.

Unitermos: Educação física; Adesão; Atividade física; Envelhecimento.

\section{Introdução}

A partir da segunda metade do século XX vem ocorrendo o fenômeno de envelhecimento da população mundial. No Brasil, a população de idosos que representava $6,0 \%$ do total de habitantes em 1980 , saltou para 7,4\% em 1991. Uma década depois já compreende $8,6 \%$ e, de acordo com o censo demográfico de 2000, a expectativa é que em 2025 haverá perto de 32 milhōes de idosos, o que representará cerca de $14 \%$ da população brasileira (IBGE, 2000). A Organização Mundial de Saúde (IBGE, 2000) presume que até 2025 o Brasil será o sexto país do mundo com o maior número de pessoas idosas.

O crescimento dessa população vem acompanhado do aumento da expectativa de vida, que passou de 66 anos, em 1991, para quase 69 anos, em 2000. Essa média é puxada para cima pelas mulheres, pois enquanto os homens vivem cerca de 65 anos, as mulheres vivem quase 73 (PASTORE, 2002). O Fundo das Naçōes Unidas Para População (FUNUAP), órgão ligado à $\mathrm{ONU}$, estima que nos países em desenvolvimento a expectativa de vida em 2025 será de 82 anos para homens e 86 para mulheres, ou seja, cerca de 21 anos a mais do que ocorre hoje em dia.

A mudança no aspecto demográfico, com a população mundial em amplo crescimento, tem levado uma parcela cada vez maior da população à perda da qualidade de vida. Tais perdas são provocadas, em parte, pelas variadas condiçóes sócioeconômicas (CAMARANO, 2003) e, também, devido à presença de doenças e a perda das capacidades físicas e funcionais, provocados pelo estilo de vida com pouca ou nenhuma atividade física. 
O idoso doente e debilitado representa aumento do custo econômico e social para o Estado, bem como, aumento da demanda do sistema de saúde. (Fris, Nomura, Ma \& Swan, 2003; Pastore, 2002). Por esta razão, existe clara preocupação em fazer com que os idosos tenham uma atitude positiva frente à atividade física, praticando-a regularmente e valorizando-a como um dos mecanismos para melhora dos aspectos biológicos, psicológicos e sociais de suas vida. Durante o IV Congresso Mundial de Atividade Física, Esporte e Envelhecimento, realizado em 1994, em Heidelberg, na Alemanha, a Organização Mundial de Saúde (OMS) endossou a atividade física regular como componente integral do envelhecimento saudável e que exercícios físicos devem fazer parte da rotina diária para a grande maioria dos idosos (CHODZKO-ZAJKO, 2000).

Apesar dos inúmeros benefícios já constatados como resultado da prática regular de atividades físicas como a melhora das funçôes biológicas, a melhora das capacidades físicas e neuromotoras, a diminuição da incidência de doenças crônicas, a melhora do funcionamento do sistema nervoso central, e o aumenta a rapidez dos processos cognitivos (SPIDURSO, 1995), a prática de atividade física ainda é uma barreira a ser superada pelos idosos.

Estudos transversais indicam um declínio na participação em atividade física dos indivíduos de idade mais avançada (GogGIO \& Morrow Junior, 2001). O estudo de SHIBATA (2000), envolvendo 671 participantes japoneses, verificou que $36 \%$ dos idosos praticam caminhada ou ginástica calistênica como forma de preservar a saúde. Nos EUA apenas15\% dos idosos se exercitam em níveis recomendados (ChodzKo-ZajKo, 2000). Já, Morey (2003) aponta dados do Departamento Norte-Americano de Saúde e Serviços Humanos, em que apenas cerca de 30\% dos homens e $15 \%$ das mulheres com idade acima de 60 anos reportaram participar regularmente de atividade física. Para LITWIN (2003), somente 38\% de uma amostra de uma comunidade Israelense está engajada em algum tipo de atividade física. MansField, MarX e GURALNIK (2003) afirmam que menos de $10 \%$ dos idosos americanos participam de alguma forma de atividade física vigorosa.

Uma análise retrospectiva da literatura acerca da taxa de adesão da população idosa aos programas de atividade física evidencia que esta não se modifica há anos, não obstante aos esforços de diversas campanhas institucionais para elevá-los, tanto em nível local como nacional, bem como dos diversos profissionais da saúde. A observação destas ações para mudar o comportamento das pessoas aponta o uso, predominante, de conteúdos informativos, o que parece significar que elas, aparentemente, não se mostram suficientemente influentes para sensibilizar a população, particularmente a idosa, a iniciar e manter-se em programas regulares de atividade física.

Com base na contradição - os efeitos da prática regular da atividade física são predominantemente benéficos e, apesar do seu reconhecimento, a população idosa continua sedentária - é que entendemos que as pessoas necessitam mais do que informaçôes para mudarem hábitos e comportamentos, o que significa que elas precisam é se educar para tais mudanças. Assim, acreditamos em um modelo educacional de educação física para que o idoso mude sua atitude e comportamento em relação à prática da atividade física, o que nos levou à criação de uma proposta de educação física para idosos com essas características.

Acreditando que um modelo educacional pode ampliar e reforçar o valor da atividade motora para que o idoso possa lidar com o processo de envelhecimento, foi desenvolvido o PAAF (Programa de Autonomia para a Prática de Atividade Física para Idosos), cuja proposta central é ensinar o participante auto gerir a própria atividade física, de modo a ter autonomia para praticá-la regular e permanentemente.

O entendimento e a compreensão dos motivos que fazem com que o idoso procure um programa de atividade física, bem como procurar saber quem é este idoso, onde mora, como vive, como está a sua saúde, qual o seu histórico de vida, entre outras, são questões fundamentais para orientar as ações e as intervençôes dos agentes que oferecem programas educacionais ou de atividade física para essa população. Além disso, permite elaborar e desenvolver estratégias que possam alcançar aqueles que possuem maior resistência à prática de atividade física. Dessa forma, neste trabalho interessa-nos verificar qual é o perfil das pessoas que ingressaram no PAAF-2002 e quais foram os principais motivos da adesão inicial. 


\section{Determinantes da adesão à prática regular de atividade física entre os idosos}

Diante da preocupação em tornar os idosos cada vez mais ativos fisicamente e com autonomia para a prática de atividades da vida diária, os interesses de pesquisadores e estudiosos estão direcionados ao conhecimento e compreensão dos fatores que os levam a praticar atividade física, tais como: quais são os tipos de atividades que eles preferem de acordo com o gênero, idade, nível de escolaridade; quais são as barreiras e os obstáculos encontrados para a prática de atividade física; quais são os principais fatores que os levam à adesão e a desistência de programas supervisionados, entre outros.

A compreensão desses fatores permitirá ao profissional de Educação Física selecionar conteúdos e elaborar estratégias mais adequadas ao público em questão, possibilitando maior identificação e interesse do aluno pelo programa. Permite ainda a economia de recursos e de tempo, visto que o risco de adotar intervenções irrelevantes e/ou inapropriadas fica reduzido.

Entre alguns modelos que surgiram a partir dos estudos da Adesão à Prática de Atividade Física temos: o modelo de aprendizagem social de comportamento de saúde (King \& MARTin, 1995); o modelo sistêmico de mudanças de comportamentos para com a saúde, composto de três fases: antecedente, adesão e manutenção (TAYlor, Miller \& FlORA, 1995); o modelo transteórico baseado na mudança de comportamento (McKowna, NAYLOR \& MCDowell, 1998).

Oкима (1997) afirma que as várias teorias desenvolvidas e utilizadas para estudar os determinantes da prática regular de atividade física são de natureza predominantemente psicológica, sendo que estes modelos isolados são inadequados para explicar a participação na atividade física. Por isso, há necessidade de modelos multidimensionais que consideram as variáveis psicológicas, ambientais e a própria atividade física de forma integrada, pois há consistentes associações entre essas variáveis. Para Bouchard, Shephard e Stephens (1993), é importante a compreensão da dissociação existente entre a adesão inicial e a manutenção da prática de atividade física, pois considera que as estratégias adotadas para a manutenção da atividade física abrangem a preparação do indivíduo para a auto-regulação do comportamento, preparando-o para possíveis recaídas. Portanto, existe necessidade de conhecer separadamente as causas da adesão, da manutenção e da desistência da prática de atividade física.
Entre os determinantes da prática regular de atividade física destacam-se: determinantes pessoais, que compreendem as características sóciodemográficas (idade, sexo, nível de escolaridade, nível sócio-econômico); determinantes ambientais (disponibilidade de tempo, local que atividade física ocorre, suporte social); determinantes da atividade física como características do programa, aspectos didáticos e a relação professor-aluno (BOUCHARD, ShepHard \& StePHens, 1995; Dishman, 1988; King \& Martin, 1995; OKUMA, 1997).

Os estudos específicos com idosos revelaram que o fator mais relevante que os levam a adotar a praticar atividade física é a preocupação com a manutenção e melhora da saúde. Embora este não seja o principal fator para mantê-los em atividade ao longo de um programa supervisionado, com certeza é o mais importante para a adesão inicial. Hivensalo, LAMPIEN e RANTANEN (1998), verificaram que $80 \%$ dos relatos dos idosos referiam-se à preocupação com a saúde como a principal razão para a adesão inicial a um programa supervisionado de atividade física. Razões sociais e psicológicas vieram logo atrás com $40 \%$ e $30 \%$ respectivamente.

Da mesma forma, Mansfield, MarX e GuralniK (2003) estudando os motivos e as barreiras para o exercício em idosos numa comunidade dos EUA, constataram que os principais motivos relatados para o engajamento de idosos na atividade física foram advertência dos médicos e obter melhor condição física. Neste estudo observaram também os fatores sociais (a presença ou fazer amigos), fatores ambientais (proximidade do local das aulas) e fatores pessoais (mais tempo e mais conhecimentos e/ou informaçōes sobre os benefícios da atividade física) como alguns motivos referidos pelos idosos para a prática de atividade física.

$\mathrm{O}$ aspecto social configura-se como outro importante fator para adesão à atividade física. Para Chogahara e YAmaguchi (1998), o suporte social está diretamente relacionado à adesão à atividade física, com a intenção de ser ativo, com a auto-eficácia e com o controle do comportamento percebido. Wenkel et al. (1994), citado por ChOGaHARA e YAMAGUCHI (1998), constataram que o suporte social da esposa ou do esposo, de outros membros da família, dos amigos e dos médicos contribuiu mais para a população de idosos se tornar ativo, do que para a população mais jovem. De acordo com 
Goggio e Morrow Junior (2001) as mulheres idosas têm maior probabilidade de aderir a programa supervisionado de atividade física devido a interação social que a atividade em grupo proporciona. Já os homens buscam benefícios para saúde.

Variáveis sócio-demográficas são importantes determinantes para a adesão atividade física. FRIIS et al. (2003) verificaram que fatores como idade, gênero, nível de escolaridade, estado civil e o estado de saúde relatado (saúde percebida) foram fundamentais para o engajamento de idosos em programa de caminhada. Segundo os autores, idosos mais jovens (entre 70 a 74 anos), os com maiores níveis de escolaridade e os maiores níveis sócio econômico têm maior probabilidade de se engajar regularmente em exercícios físicos do que os mais velhos (acima de 80 anos) e os com menor nível escolar e de renda.

Neste estudo, verificaram também que os idosos viúvos, divorciados e solteiros também tende a participar mais de atividade física e de caminhadas do que os casados. Para eles, o idoso casado dificilmente se engaja na atividade física se o cônjuge não estiver envolvido, criando assim uma barreira para a prática regular de exercícios. Resultados semelhantes foram encontrados por LITWIN (2003), que verificou que o nível sócio-econômico, o nível de educação e a saúde percebida de idosos Israelense foram associados positivamente para prática regular de atividade física.

O estado de saúde e a saúde percebida são aspectos importantes, pois influenciam muito os aspectos afetivos como auto-eficácia e a intenção de ser fisicamente ativo. De acordo com FriIs et al. (2003), as pessoas que se percebem com saúde muito boa ou excelente são mais prováveis a engajar em sessões de caminhadas do que os com saúde percebida negativa. Mansfield, MarX e GURALNIK (2003) verificaram que problemas de saúde e presença de dores crônicas foram as principais barreiras percebidas para a não participação de idosos em exercícios e caminhadas. LEVEILLE, MANSFIELD e GURALNIK (2003), estudando idosos de 75 a 85 anos constataram que $43 \%$ da sua amostra relataram presença de dores musculo-esqueléticas crônicas. Segundo os autores, a ocorrência de dores está diretamente ligada auto-eficácia negativa, influenciando a atitude para o exercício, podendo levar ao comportamento sedentário.

Hivensalo, Lampien e Rantanen (1998) verificaram que cerca de $85 \%$ de idosos atribuíram seus sedentarismos ao estado de saúde que consideraram pobre. No mesmo estudo, estes autores verificaram que os principais obstáculos encontrados para a não participação em aulas de exercícios supervisionados foram: falta de interesse, falta de amigos e companhia para exercitar-se. Entre as barreiras para o exercício físico, encontradas no estudo de MANSFILED, MarX e GURALNIK (2003) temos: problemas de saúde e a ocorrência de dores, preguiça, a falta de tempo e a presença de fadiga. CHOGAHARA e YAMAGUCHI (1998) verificaram que as barreiras pessoais frequentemente citadas como "eu estou muito velho" ou "atividade física é muito arriscada", são alguns dos motivos que levam o idoso à não adesão.

Um ponto importante que deve ser observado com atenção diz respeito às preferências que o idoso tem pelas formas de atividade física. Embora as aulas formais de programas supervisionados sejam as principais formas de intervenção, a maioria dos idosos, sobretudo os do sexo masculino, apreciam mais atividades realizadas individualmente, em detrimento das aulas formais em grupo. Esses achados são válidos pelo menos para os estudos de Mills, Stewart, Sepsis e King (1997) e de Wilcox, King e Brassington (1999).

Segundo Mills et al. (1997), há um crescente aumento na preferência por atividades individuais em casa. Da mesma forma, Wilcox, King e BRASSINGTON (1999) verificaram que a maioria dos idosos que fez parte de sua amostra demonstrou preferências por exercícios individuais. Os números de seus achados revelaram que $85 \%$ dos idosos do sexo masculino têm preferência por exercícios individuais. Entre as mulheres cerca de 59\% com idade entre 57 a 64 anos preferem se exercitar sozinhas.

Diante desses achados, com a maioria dos idosos preferindo atividades individuais e em casa, Mills et al. (1997) afirmam que esses indivíduos são menos prováveis a prestar atenção em aulas formais de ginástica em programas supervisionados. Dessa forma, esse grupo possui grande probabilidade de desistência. Wilcox, King e Brassington (1999) sugerem que os programas comunitários enfatizem nos seus conteúdos, atividades físicas que os idosos possam realizar fora da aula. Esses programas, na visão dos autores, provavelmente serão mais eficientes ao proporcionarem instruções iniciais sobre as atividades físicas e realizar um acompanhamento periódico junto ao participante.

Não podemos negar a importância desses estudos para compreendermos mais sobre os motivos que levam o idoso a participar de programas de atividade física. No entanto, devemos questionar se esse tipo de comportamento, apresentados pelos dois estudos anteriores, pode ser estendido aos diversos povos e culturas. Talvez a preferência por exercícios individuais 
seja restrita às populações cujas amostras foram extraídas. Como exemplo de trabalho que contradiz os anteriores, podemos citar os estudos realizados com a população japonesa, quando CHOGAHARA e YAMAGUCHI (1998) afirmam que mais de 65\% dos idosos que se exercitam regularmente preferem realizá-lo em grupo, a praticar individualmente. Goggio e Morrow Junior (2001) sugerem que programa para mulheres deve focalizar a interação social e a rede social, como fazer novas amizades, pois segundo os autores as mulheres buscam principalmente interagir socialmente.

Outro importante fator que leva o idoso a praticar atividade física regular, individualmente ou grupo, e que merece ser destacado, está relacionado ao histórico de vida das pessoas.

Geralmente, o indivíduo que foi fisicamente ativo durante sua juventude e/ou na meia idade, está mais propenso a se tornar, ou melhor, a continuar ativo na velhice. Baseados na Teoria da Continuidade, LANGLEY e KNIGTH (1999) verificaram que o sucesso no envolvimento esportivo ao longo da vida serviu tanto como alicerce para o envolvimento em atividade física, quanto para imediata adaptação ao processo de envelhecimento. Neste estudo, os autores concluem que a natureza competitiva, adicionada aos seus desejos por interelações sociais, foi importante para as pessoas mediarem os aspectos das mudanças no processo de envelhecimento.

Para Hivensalo, Lampien e Rantanen (1998), a história de vida pode influenciar na motivação individual para o envolvimento com a atividade física. Eles citam como exemplo o indivíduo que ao longo de sua vida adulta dedicou seu tempo quase que exclusivamente ao trabalho. Provavelmente, essa pessoa ficou privada de realizar exercícios físicos na juventude, sendo que as habilidades e as capacidades necessárias para realização de atividades físicas não foram suficientemente desenvolvidas. Assim, sua velhice provavelmente será marcada pelo sedentarismo.

Curtis, White e McPherson (2000) afirmam que a vida do idoso é uma continuação de um passado pessoal, cujo estilo de vida escolhido na velhice será o mais parecido com padrão de vida que ele viveu na meia-idade. Mansfiled, MarX e GURALNIK (2003) verificaram que indivíduos que não participaram de atividade física no passado tende a ter maior atitude negativa para o exercício do que os idosos com histórico de atividade física.
Embora observemos um tímido aumento no nível de atividade física entre a população idosa, ainda não é possível explicar exatamente quais são os principais motivos da adesão, da manutenção e desistência. Curtis, White e McPherson (2000) afirmam que as tradicionais teorias gerontológicas não podem explicar os motivos do aumento da atividade física para o idoso.

Em recente trabalho longitudinal os autores acompanharam a participação de uma amostra da população canadense em atividade física, constatando um aumento do nível de atividade física em todos os subgrupos ao longo do período estudado (1981 a 1988). Neste estudo Curtis, White e McPherson (2000) recorreram à Teoria da Ressocialização para explicam o fato do grupo que mais aumentou seu nível de atividade física no período de oito anos (1981 a 1989) foi o de homens com mais de 65 anos.

Segundo Chogahara e Yamaguchi (1998), a Teoria da Resocialização é utilizada para explicar mudanças fundamentais ou transições comportamentais ou cognitivas, que proporcionam ao indivíduo experiências que possibilitem mudanças em suas crenças, conhecimentos e valores. Envolve básica e rápida mudança no estilo de vida como novo "status" social, viuvez ou aposentadoria.

Chogahara e Yamaguchi (1998) consideram importante identificar os indivíduos que se enquadram na Teoria da Resocialização, pessoas que passaram por recentes mudanças nas suas vidas e que decidiram optar por uma vida mais ativa, e os que se enquadram na Teoria da Continuidade, ou seja, aqueles que sempre foram ativos ao longo de suas vidas. De acordo com as proposições da Teoria da Continuidade, a pessoa escolherá o estilo de vida mais parecido com padróes que ela viveu na juventude. Para LANGLEY e KNIGTH (1999), a continuidade refere-se à consistência de um padrão de açôes sobre diferentes estágios da vida.

Indivíduos que foram ativos ao longo da vida possuem um alto nível de percepção sobre a eficácia de um programa de atividade física. Além disso, têm maior probabilidade de possuir preferências por determinadas atividades. Ao contrário, os que foram sedentários na juventude e meia-idade, têm atitude negativa diante da atividade física. Disso decorre a importância de elaborar estratégias diferentes para aqueles que são previamente sedentários e para aqueles que têm sido continuamente envolvido com a atividade física. 


\section{Método}

Fizeram parte deste estudo 14 homens (26\%) e 40 mulheres $(74 \%)$, num total de 54 pessoas, com idade acima de 60 anos e que foram selecionados por sorteio para ingressar, em 2002, no Programa Autonomia para Atividade Física para Idosos - PAAF, um serviço de extensão comunitário oferecido pela Escola de Educação Física e Esporte da Universidade de São Paulo - EEFEUSP e desenvolvido pelo Grupo de Estudo e Pesquisa de Programas de Educação Física para Idosos (GREPREFI).

Todos os dados foram coletados antes do início do PAAF 2002.

Para verificar o perfil sócio demográfico dos sujeitos foi utilizado um questionário, constituído por questões abertas e fechadas, em que foram consideradas as seguintes variáveis: genêro, idade, nível de escolaridade, estado civil; saúde percebida do ingressante; a atividade profissional e a atividade física pregressa.

O nível sócio-econômico foi obtido pela classificação da Associação Brasileira dos Institutos de Pesquisas de Mercado (ABIPEME).

Para verificar os motivos da adesão inicial ao PAAF foi utilizada uma tabela do tipo Liekert, onde foram elencados onze objetivos, que poderiam ter influenciado os alunos a iniciar no programa. Os ingressantes deveriam atribuir valores que variavam de zero (para o menos importante) a quatro (para os mais importantes) para cada um dos objetivos apresentados na TABELA.

Também foi feita uma questão aberta para que os sujeitos indicassem o objetivo que pretendiam alcançar no PAAF. Aqueles com dificuldades no preenchimento do questionário foram auxiliados pelos professores, que não interferiram nas respostas dos alunos, apenas escreveram literalmente suas falas.
A análise das variáveis referentes ao perfil sóciodemográfico (gênero, idade, escolaridade, nível sócioeconômico, estado civil) foi feita através da estatística descritiva, representada pelo percentual das respostas. Já a análise dos motivos de adesão foi feita pelo Índice de Consistência Interna de Liekert, pelo Coeficiente de Concordância de Kendall (W) eTeste de Friedman $\left(\mathrm{Xr}^{2}\right)$, sendo adotado como nível de significância $(p=0,05)$.

Para determinar o nível de significância das respostas dos alunos, realizamos o cálculo do Coeficiente de Concordância de Kendall (W) com p = 0,05. Neste teste obtivemos $\mathrm{p}<0,001$ para $\mathrm{gl}=(\mathrm{N}-1)$. Este resultado indica que há concordância entre os alunos, no que se refere à atribuição de importância aos objetivos apresentados a eles. Com isto, podemos considerar que a ordem estabelecida pelos alunos, por meio da escala de Lierket, é representativa para nossa amostra.

Ainda, aplicamos o Teste de Friedman $\left(\mathrm{Xr}^{2}\right)$ para $\mathrm{p}=0,05$ e obtivemos o nível de significância (p) entre 0,02 e 0,05 , para $\mathrm{gl}=10$. Isto significa que a classificação dos objetivos mais importantes e dos menos importantes não foi mero acaso. Portanto podemos considerar verdadeira e estatisticamente significante a classificação obtida pelo Índice de Consistência Interna de Liekert.

A análise do objetivo almejado pelo sujeito, levantada pela questão aberta, foi feita através da frequência de respostas que determinaram sete categorias:

- melhorar o condicionamento físico;

- adquirir conhecimentos;

- melhorar a saúde;

- melhorar a qualidade de vida;

- aspectos psicológicos e emocionais,

- interação social/fazer amizades;

- ocupar o tempo livre.

\section{Resultados}

Em relação à idade dos sujeitos (TABELA 1), observa-se que a idade média era de 66,45 anos $(5,176)$. A maioria $53,70 \%$ corresponde à faixa etária dos 60 ao 65 anos $(57,14 \%$ dos homens e $52,5 \%$ das mulheres); $24,07 \%$ pertence à faixa etária que vai dos 66 a 70 anos $(14,29 \%$ dos homens e $27,5 \%$ das mulheres); $16,67 \%$ dos 71 a 75 anos (21,43\% dos homens e $15 \%$ das mulheres), 3,7 \% dos 76 aos 80 anos (um homens e uma mulher) e $1,85 \%$ acima de 81 anos (uma mulher).
Outra característica dos sujeitos que ingressaram no PAAF diz respeito ao gênero (TABELA 1), em que se nota que a grande maioria era do sexo feminino $(74 \%)$, sendo apenas $26 \%$ de homens, confirmando o que se tem observado em programas para idosos.

Com relação ao nível de escolaridade dos sujeitos, a TABELA 2 mostra que $42,59 \%$ estudaram até o nível superior $(71,5 \%$ dos homens e $32,5 \%$ das mulheres), 22,22\% dos ingressantes cursaram até o segundo grau $(21,43 \%$ dos homens e $22,5 \%$ das 
mulheres). Quanto aos demais sujeitos, 16,67\% completaram o primeiro grau $(7,14 \%$ dos homens e $20,0 \%$ das mulheres), outros $14,81 \%$ não concluíram o primeiro grau e 3,7\% não possuíam nenhuma escolarização, sendo as duas últimas condições referentes ao sexo feminino.

TABELA 1 - Idade e gênero dos ingressantes do PAAF-2002.

\begin{tabular}{|c|c|c|c|c|c|c|c|c|c|c|c|c|}
\hline & \multicolumn{2}{|c|}{60 a 65 anos } & \multicolumn{2}{|c|}{66 a 70 anos } & \multicolumn{2}{|c|}{71 a 75 anos } & \multicolumn{2}{|c|}{76 a 80 anos } & \multicolumn{2}{|c|}{ Acima de 81 anos } & \multicolumn{2}{|c|}{ Total } \\
\hline & Total & $(\%)$ & Total & $(\%)$ & Total & $(\%)$ & Total & $(\%)$ & Total & $(\%)$ & Total & $(\%)$ \\
\hline Masculino & 8 & 57,14 & 2 & 14,29 & 3 & 21,43 & 1 & 7,14 & 0 & 0 & 14 & 25,93 \\
\hline Feminino & 21 & 52,50 & 11 & 27,5 & 6 & 15,0 & 1 & 2,5 & 1 & 2,5 & 40 & 74,07 \\
\hline Total & 29 & 53,70 & 13 & 24,07 & 9 & 16,67 & 2 & 3,7 & 1 & 1,87 & 54 & 100 \\
\hline
\end{tabular}

TABELA 2 - Nível de escolaridade dos ingressantes do PAAF, por gênero.

\begin{tabular}{lcccccccccc}
\hline & \multicolumn{2}{c}{ Sem escolaridade } & \multicolumn{2}{c}{ Primário } & \multicolumn{2}{c}{ Ginásio } & \multicolumn{2}{c}{ Colegial } & \multicolumn{2}{c}{ Superior } \\
\cline { 2 - 10 } & Total & $(\%)$ & Total & $(\%)$ & Total & $(\%)$ & Total & $(\%)$ & Total & $(\%)$ \\
\hline Masculino & 0 & 0 & 0 & 0 & 1 & 7,14 & 3 & 21,43 & 10 & 71,43 \\
Feminino & 2 & 5,0 & 8 & 20,0 & 8 & 20,0 & 9 & 22,5 & 13 & 32,50 \\
Total & 2 & 3,7 & 8 & 14,81 & 9 & 16,67 & 12 & 22,22 & 23 & 42,59 \\
\hline
\end{tabular}

Quanto ao nível sócio-econômico dos sujeitos nota-se, na TABELA 3, que 47,06\% foram classificados, de acordo com $\mathrm{ABIPEME}$, nos níveis $\mathrm{A}\left(\mathrm{A}_{1}=\right.$ $3,92 \%$ e $\left.A_{2}=43,14 \%\right), 43 \%$ nos níveis $B(23,53 \%=$ $\mathrm{B}_{1}$ e $17,65 \%-\mathrm{B}_{2}$ ) e apenas $11,76 \%$ foram classificados no nível C. Nenhum ingressante foi classificado nos níveis sócio-econômico $\mathrm{D}$ e $\mathrm{E}$.

TABELA 3 - Nível sócio-econômico dos ingressantes do PAAF-2002 de acordo com ABIPEME.

\begin{tabular}{lccccc}
\hline Nível sócio-econômico & $\mathbf{A}_{1}$ & $\mathbf{A}_{2}$ & $\mathbf{B}_{1}$ & $\mathbf{B}_{2}$ & $\mathbf{C}$ \\
\hline Número de alunos & 2 & 22 & 12 & 9 & 6 \\
$\%$ & 3,92 & 43,14 & 23,53 & 17,65 & 11,76 \\
\hline
\end{tabular}

Quando se faz análise da atividade profissional dos sujeitos, exercida ao longo da vida (TABELA 4), observa-se que a grande maioria (83\%) desempenhou alguma atividade profissional, o que, em relação ao sexo masculino, é esperado. Entretanto, o que merece ser destacado é o perfil profissional das mulheres deste estudo, em que se observa que a grande maioria delas, ou seja, 77,5\% mantiveram atividades profissionais ao longo da vida, com apenas $22 \%$ tendo sido exclusivamente donas de casa.
TABELA 4 - Atividades profissionais exercidas pelos alunos do PAAF.

\begin{tabular}{lccc}
\hline \multicolumn{1}{c}{ Categorias } & Homens & Mulheres & Total \\
\hline Profissional liberal & 7 & 4 & 11 \\
Empresário & 2 & 0 & 2 \\
Trab. comércio & 0 & 4 & 4 \\
Magistério & 1 & 11 & 12 \\
Func. público & 0 & 2 & 2 \\
Serv. bancário & 1 & 2 & 3 \\
Militar & 2 & 0 & 2 \\
Secretária exec. & 0 & 1 & 1 \\
Dona de casa & 0 & 9 & 9 \\
Outros (costureira, & 1 & 7 & 8 \\
encanador, copeira) & & 40 & 54 \\
Total & 14 & & \\
\hline
\end{tabular}

Em relação ao perfil de saúde dos ingressantes do PAAF-2002, a TABELA 5 mostra que apenas dois sujeitos não apresentavam nenhuma doença, sendo ambos do sexo masculino. Os demais (96\%) apresentaram uma ou mais doenças, evidenciando que as mulheres apresentavam maior quantidade de doenças do que os homens. 
TABELA 5 - Quantidade de doenças relatadas.

\begin{tabular}{lcccccccc}
\hline $\begin{array}{c}\text { Quantidade de } \\
\text { doenças relatadas }\end{array}$ & $\begin{array}{c}\text { Sem } \\
\text { doenças }\end{array}$ & $\mathbf{1}$ & $\mathbf{2}$ & $\mathbf{3}$ & $\mathbf{4}$ & $\mathbf{5}$ & $\mathbf{6}$ & $\mathbf{7}$ \\
\hline Feminino & 0 & 5 & 5 & 11 & 7 & 8 & 2 & 2 \\
Masculino & 2 & 2 & 8 & 1 & 0 & 1 & 0 & 0 \\
Total & 2 & 7 & 13 & 12 & 7 & 9 & 2 & 2 \\
\hline
\end{tabular}

Utilizando-se a classificação sugerida por SHEPHARD (1997), doenças do sistema cardiorespiratório, doenças músculo-esquelética, doenças metabólicas e doenças do sistema nervoso central (TABELA 6), observa-se que o problema de saúde mais relatado pelos homens neste estudo refere-se ao sistema cardiorespiratório, sendo a hipertensão arterial a mais frequente, apontada por $57 \%$ deles. Em seguida aparecem as doenças do sistema metabólico com $21,4 \%$ dos sujeitos relatando algum tipo de dislipidemia e apenas um relato de diabetes e um de câncer. Quanto às doenças músculo-articulares nota-se apenas um relato de problemas articulares e nenhum para o sistema nervoso.

Já, no caso das mulheres também predominou a presença de doenças do sistema cardiorespiratório, com $57,5 \%$ relatando ter hipertensão, seguida de doenças do sistema metabólico, com $35 \%$ relatando ter dislipidemia e $20 \%$ diabetes. Também se observa que $27,5 \%$ relataram problemas do sistema nervoso, cuja principal ocorrência se referiu à labirintite.

TABELA 6 - Presença de doenças relatadas pelos ingressantes do PAAF-2002 de acordo com a classificação de SHEPHARD (1997).

\begin{tabular}{lcccccccc}
\hline Tipos de doenças & \multicolumn{2}{c}{$\begin{array}{c}\text { Doenças Sist. Cardior- } \\
\text { respiratório }\end{array}$} & \multicolumn{2}{c}{$\begin{array}{c}\text { Doenças Musculo- } \\
\text { esqueléticas }\end{array}$} & \multicolumn{5}{c}{ Doenças Metabólicas Doenças Sist. Nervoso } \\
\hline Sexo & Masc. & Fem. & Masc. & Fem. & Masc. & Fem. & Masc. & Fem. \\
Número de alunos & 8 & 23 & 3 & 36 & 8 & 20 & 0 & 16 \\
$\%$ & 57 & 57,5 & 21,4 & 90 & 57 & 50 & 0 & 40 \\
\hline
\end{tabular}

Mais uma variável analisada neste trabalho referese às experiências com atividade física vivida antes dos sujeitos ingressarem no PAAF. A TABELA 7 mostra que outra característica interessante dos ingressantes é que a grande maioria já era fisicamente ativa. Verificando a atividade física que praticavam até o momento em que o programa iniciou, constatou-se que $81,5 \%$ realizavam pelo menos um tipo de atividade física e apenas 18,5\% não praticavam nenhuma atividade física até ingressar no PAAF.

TABELA 7 - Histórico de atividade física dos ingressantes do PAAF-2002 (Da infância até os 60 anos de idade).

\begin{tabular}{|c|c|c|c|c|c|c|}
\hline \multirow{2}{*}{ Número de atividade } & \multicolumn{2}{|c|}{ Masculino } & \multicolumn{2}{|c|}{ Feminino } & \multicolumn{2}{|c|}{ Total } \\
\hline & Total & $(\%)$ & Total & $(\%)$ & Total & $(\%)$ \\
\hline 0 & 1 & 7,14 & 9 & 22,5 & 10 & 18,52 \\
\hline 1 & 8 & 54,14 & 12 & 30,0 & 20 & 37,04 \\
\hline 2 & 3 & 21,43 & 9 & 22,5 & 12 & 22,22 \\
\hline 3 & 1 & 7,14 & 7 & 17,5 & 8 & 14,81 \\
\hline 4 & 1 & 7,14 & 3 & 7,5 & 4 & $7,41 \%$ \\
\hline Total & 14 & & 40 & & 54 & \\
\hline
\end{tabular}

Analisando a atividade física pregressa dos sujeitos (TABELA 8), verificamos que 66,66\% dos ingressantes do PAAF-2002 (71,45\% dos homens e 65\% das mulheres) eram fisicamente ativos. Podemos observar que $24,81 \%$ foram fisicamente ativos desde a infância (três homens e cinco mulheres). Outros $51,85 \%$ iniciaram-na em outras fases da vida: 1,85\% (um homem) iniciou na adolescência;
$1,85 \%$ (um homem) aos 20 anos; 18,52\% (dois homens e oito mulheres) aos 30 anos; $11,11 \%$ (dois homens e quatro mulheres) aos 40 anos; e 18,52\% (um homem e nove mulheres) iniciaram aos 50 anos, mantendo-se todos ativos até o início do PAAF 2002.

Considerando somente os indivíduos fisicamente ativos, percebemos que as mulheres iniciaram a 
prática de atividade física mais tardiamente, quando comparadas aos homens. Entre as 26 ingressantes do PAAF-2002 que eram fisicamente ativas, 19,23\% sempre foram ativas desde a infância. As outras 80,76\% começaram a praticar atividade física após os 30, 40 ou 50 anos de idade. Já entre os homens que eram fisicamente ativos exatamente $50 \%$ iniciaram suas atividades até os 20 anos de idades. Os demais iniciaram aos 30 anos (20\%), aos 40 anos (20\%) e aos 50 anos (10\%).

$\mathrm{Na}$ TABELA 9 observamos que havia 18 pessoas $(33,33 \%)$, sendo quatro homens e 14 mulheres que foram considerados sedentários. Entre os indivíduos sedentários que ingressaram no PAAF-2002, 9,26\% (três homens e duas mulheres) mantiveram atividade física somente até a adolescência; 1,85\% (uma mulher) praticou até os 20 anos de idade e $3,70 \%$ (duas mulheres) praticou até os trinta anos de idade, mantendo-se sedentários até entrarem no PAAF. Vale destacar que apenas 18,5\% dos alunos (um homem e nove mulheres) foram sedentários ao longo de toda a vida. Ou seja, nunca praticou atividade física até o seu ingresso no PAAF-2002.

TABELA 8 - Fase da vida que o ingressante do PAAF-2005 iniciou a prática de atividade física.

\begin{tabular}{lcccccc}
\hline \multirow{2}{*}{ Período da vida que iniciou a atividade física } & \multicolumn{2}{c}{ Masculino } & \multicolumn{2}{c}{ Feminino } & \multicolumn{2}{c}{ Total } \\
\cline { 2 - 7 } & Total & $(\%)$ & Total & $(\%)$ & Total & $(\%)$ \\
\hline Infância & 3 & 21,47 & 5 & 12,5 & 8 & 14,81 \\
Adolescência aos 20 anos & 1 & 7,14 & 0 & 0 & 1 & 1,85 \\
Dos 20 aos 30 anos & 1 & 7,14 & 0 & 0 & 1 & 1,85 \\
Dos 30 aos 40 anos & 2 & 14,28 & 8 & 20,0 & 10 & 18,52 \\
Dos 40 aos 50 anos & 2 & 14,28 & 4 & 10,0 & 6 & 11,11 \\
Dos 50 aos 60 anos & 1 & 7,14 & 9 & 22,5 & 10 & 18,52 \\
Total & 10 & 71,45 & 26 & 65,0 & 36 & 66,66 \\
\hline
\end{tabular}

TABELA 9 - Fase da vida em que houve a interrupção da atividade física.

\begin{tabular}{lcccccc}
\hline \multirow{2}{*}{ Período que interrompeu a atividade física } & \multicolumn{2}{c}{ Masculino } & \multicolumn{2}{c}{ Feminino } & \multicolumn{2}{c}{ Total } \\
\cline { 2 - 7 } & Total & $(\%)$ & Total & $(\%)$ & Total & $(\%)$ \\
\hline Infância & 3 & 21,43 & 2 & 5 & 5 & 9,26 \\
Adolescência & 0 & 0 & 1 & 2,5 & 1 & 1,85 \\
Até os 20 anos de idade & 0 & 0 & 2 & 5 & 2 & 3,70 \\
Sempre sedentário & 1 & 7,14 & 9 & 22,5 & 10 & 18,51 \\
Total & 4 & 28,52 & 14 & 35 & 18 & 33,33 \\
\hline
\end{tabular}

\section{Os motivos de adesão inicial ao PAAF-2002}

Um forte determinante para a adesão a programas supervisionados de atividade física é a expectativa do aluno em relação ao programa que está iniciando. Por isso, procuramos saber quais os objetivos que desejavam alcançar no PAAF 2002. Para tanto, foi apresentado tabela tipo Liekert com 11 objetivos para que o ingressante atribuísse valores de zero (sem importância) a quatro (muito importante).

Para classificação dos objetivos mais importante, segundo os ingressantes, foi calculado o Índice de Consistência Interna de Liekert, que nos revelou a ordem de importância expressa na TABELA 10.
TABELA 10 - Classificação dos objetivos de acordo com o ÍndicedeConsistência Interna deLiekert-ICI.

\begin{tabular}{llc}
\hline & \multicolumn{1}{c}{ Categorias } & (ICI) \\
\hline 1 & Aprender fazer exercícios corretamente. & 0,2 \\
2 & Melhorar condição física e funcional. & 0,333 \\
3 & Prevenir problemas de saúde. & 0,667 \\
4 & Perder peso. & 0,933 \\
5 & Aumentar bem estar emocional. & 0,933 \\
6 & Interagir com outras pessoas. & 1,07 \\
7 & Ampliar rede de amigos. & 1,467 \\
8 & Aprender lidar com problemas de saúde. & 2,2 \\
9 & Melhorar performance esportiva. & 2,467 \\
10 & Ocupar o tempo livre. & 2,867 \\
\hline
\end{tabular}


Para confirmar a ordem estabelecida pelo Índice de Consistência Interna de Liekert, verificamos o nível de significância das respostas dos alunos por meio do Teste de Coeficiente de Concordância de Kendall (W) com p $=0,05$. Neste teste obtivemos $\mathrm{p}<0,001$ para $\mathrm{gl}=(\mathrm{N}$ 1). Este resultado indica que houve concordância entre os alunos no que se refere à atribuição da importância dos objetivos. Portanto, podemos considerar como verdadeira a ordem estabelecida pelo calculo do índice de consistência interna de Liekert (ICI).

Aplicamos ainda o Teste de Friedman $\left(\mathrm{Xr}^{2}\right)$ para $\mathrm{p}=$ 0,05 , obtendo nível de significância (p) entre 0,02 e 0,05 , para $\mathrm{gl}=10$, o que significa que podemos considerar que a classificação dos objetivos mais importantes e os menos importantes não foi mero acaso. Portanto, podemos considerar verdadeira e estatisticamente significante a classificação apresentada na TABELA 10.

Ainda para verificar os objetivos que os alunos ingressantes procuram no PAAF, foi feita uma questão aberta para que os sujeitos indicassem o objetivo que pretendia alcançar. Esta questão visava

\section{Discussão}

A grande concentração de sujeitos nos sub-grupos etários mais novos determina o primeiro destaque a ser feito para o perfil dos iniciantes do PAAF 2002, ou seja, a maioria deles tinha entre 60 e 65 anos de idade, sendo $57 \%$ do sexo masculino e $52,5 \%$ do feminino, com média de idade de 62,4 anos $( \pm 1,64)$ e entre os 66 aos 70 anos $(68,3$ anos $\pm 1,43)$, totalizando $78 \%$ dos sujeitos entre os 60 e 70 anos, caracterizando um público composto principalmente de idosos jovens. Estes resultados vão ao encontro daqueles reportado pela literatura, confirmando que são os idosos mais jovens aqueles que tendem a iniciar ou a participar de programas de atividade física.

No estudo de FriIs et al. (2003) foi constatado que idosos mais jovens, com níveis de escolaridade e sócio-econômico mais altos têm maior probabilidade de se engajar em atividade física do que idosos mais velhos e com níveis de escolaridade e de renda mais baixos. Resultados semelhantes foram encontrados também por LiTWIN (2003), ao estudar idosos de uma comunidade israelense.

Com relação ao gênero, alguns estudos indicam que as mulheres são mais propensas a participar de programas supervisionados de atividade física, dando maior importância ao trabalho em grupo do que os homens (GOGGIO \& MORROW JUNIOR, 2001; WIL- o confronto entre as respostas da questão aberta e as da tabela, reforçando-a ou não. Além disso, permite maior liberdade e espontaneidade do aluno para respondê-la, evitando que seja direcionado por respostas pré-estabelecidas, como as da tabela tipo Liekert. A TABELA 11 revela os objetivos mais citados pelos alunos.

TABELA 11 - Objetivos que apareceram com maior frequência nas respostas dos ingressantes do PAAF-2002 à questão aberta.

\begin{tabular}{|c|c|c|}
\hline Categorização dos objetivos & $\begin{array}{l}\text { Número } \\
\text { de alunos }\end{array}$ & $\%$ \\
\hline Melhorar condicionamento físico & 22 & 30 \\
\hline Adquirir conhecimentos & 15 & 20 \\
\hline Melhorar saúde & 14 & 19 \\
\hline Melhorar qualidade de vida & 8 & 11 \\
\hline Aspectos psicológico/emocional & 7 & 9 \\
\hline Interação social/fazer amizades & 5 & 7 \\
\hline Ocupar tempo livre & 3 & 4 \\
\hline
\end{tabular}

COX, King \& Brassington, 1999). Adiciona-se a isso, o fato de que as mulheres são as que mais participam de atividades extradoméstica como organização de movimentos populares, cursos especiais e viagens (CAMARANO, 2003).

Esse processo de feminilização nos grupos de idosos não acontece somente devido à predominância de mulheres entre as pessoas com mais de 60 anos. De acordo com BARBOSA (2003), a forma como o homem interage com seu processo de envelhecimento e como se coloca nos grupos, se desenvolve a partir das representações de masculinidade presentes na sociedade. Isso leva, segundo a autora, a menor participação dos homens nos grupos de convivências de idosos.

Os sujeitos que ingressaram no PAAF-2002 apresentaram níveis muito elevados de escolarização, com média de 12,79 anos de estudo, principalmente se comparados com os dados do último Censo Demográfico de 2000, cuja média é de 3,4 anos de estudo para a população de idosos, sendo que as mulheres apresentam menor nível de escolarização do que os homens idosos (3,1 anos para as mulheres, contra 3,5 anos de estudo entre os homens). Já no Estado de São Paulo, de acordo com o IBGE (2000), a média de anos que o idoso estudou é de 4,4 anos, sendo que entre os homens a média é de 4,7 anos e entre as mulheres é de 3,8 anos de estudo. 
De acordo com PASTORE (2002) as mulheres idosas apresentam nível de escolaridade inferior ao dos homens. Baseado no censo de 2000, ele afirma que entre as mulheres com idade acima de 70 anos de idades, $43 \%$ são analfabetas, contra $38 \%$ dos homens de mesma idade. Das que possuem maiores níveis de escolaridade, a maioria não atingiu o antigo primário (atual ensino fundamental-I).

Entre os ingressantes do PAAF-2002 verificamos que os homens possuem mais tempo de escolaridade do que as mulheres. A média feminina foi de 9,75 anos de estudos, ao passo que entre os homens foi de 13,64 anos. Embora esses resultados confirmam a diferença entre homens e mulheres que o Censo de 2000 constatou, verificamos que as mulheres que fizeram parte deste estudo possuíam muito mais tempo de estudo do que a média da população de mulheres idosa. Entre as que possuem maiores níveis de escolaridade, segundo o Censo Demográfico de 2000, a maioria não completou o antigo curso primário (quatro anos de escola), apresentando uma média de 2,4 anos de estudo.

A alta escolarização observada neste estudo confirma que esta é uma característica marcante do perfil dos ingressantes do PAAF, corroborando nossas observações desde que ele foi implantado, e vai ao encontro do que foi visto na literatura no que se refere ao idoso praticante de atividade física.

Os egressos do PAAF-2002 possuíam um poder aquisitivo de médio para alto, sobretudo, se o compararmos com o rendimento médio dos idosos da população brasileira, que é de 657 reais (IBGE, 2000). No Estado de São Paulo a renda média mensal, segundo o IBGE (2000), é de 893,00 reais, sendo que na zona rural é 531,00 reais e na zona urbana, 913,00 reais, o que indica níveis elevados de renda entre os ingressantes do PAAF-2002.

Como se pode notar, os sujeitos deste estudo tinham como característica serem idosos jovens, com alto nível sócio-econômico e de escolaridade, o que vai ao encontro com o que foi visto na literatura. Estudos como o de FriIs et al. (2003) e LITWIN (2003) demonstraram que os idosos jovens, com maiores níveis de escolaridade e com maiores níveis sócio-econômico são aqueles com maiores probabilidades de participarem de programas de atividades física.

O que chamou bastante a atenção foi o fato que a maioria das mulheres egressas do PAAF2002 desempenhou alguma atividade profissional, contrariando estudos como o de CAMARANO (2003), cuja maioria das idosas brasileiras da atualidade não exerceu trabalho remunerado durante sua vida, o que não se confirmou para as mulheres desta pesquisa.

GIATTI e BARRETO (2002) identificaram um grande percentual de mulheres idosas que não trabalhavam e não eram aposentadas. Isto indica a dedicação feminina às atividades de cuidado com a casa e a família, que era o papel predominante das mulheres da geração nascidas até 1933. A participação das mulheres no mercado de trabalho sempre foi inferior à dos homens. De acordo com as autoras, este fenômeno é o reflexo da feminização do trabalho iniciado nos anos 70. Segundo as autoras, as mulheres que trabalharam fora de casa apresentam menor incidência de doenças crônicas e melhores condiçōes físicas.

Esses resultados apontam um aspecto extremamente importante, que pode trazer à tona mais uma peculiaridade no perfil das mulheres que procuram programas supervisionados de atividade física. Parece que aquelas que sempre trabalharam fora de casa têm maior interesse ou motivação para a prática de atividade física, muito embora não haja nenhum trabalho na literatura que possa evidenciar essa suposição. No entanto, nos PAAFs anteriores já observamos esta peculiaridade das mulheres que ingressam no programa.

Nossa hipótese talvez possa ser corroborada pelo fato que houve grande número de desistentes entre as donas de casa. Observamos que dos $22 \%$ (9 donas de casa) que iniciaram o PAAF, quase a metade $(44,5 \%)$ não chegou ao seu final, sendo que apenas uma delas desistiu por motivo de saúde. As demais não justificaram o motivo da desistência.

Além disso, as mulheres que trabalharam fora foram muito mais ativas durante sua vida do que as que sempre foram donas de casa. Para nossa amostra feminina houve forte relação entre o nível de escolaridade e a prática de atividade física. Mulheres com alto nível de escolaridade (colegial ou curso superior) foram mais ativas do que as com baixo nível de escolaridade ( $\mathrm{p}=$ 0,0295 para o Teste de Fisher, inferior ao $\mathrm{p}=0,05$ ).

Já com relação à saúde percebida, de acordo com CAMARANO (2003), as mulheres apresentam maiores chances do que os homens de desenvolverem as doenças típicas do envelhecimento, o que se verificou neste estudo. Todas relataram possuir algum tipo de doença, sendo que em $75 \%$ delas há presença de três ou mais doenças, ao passo que entre os homens apenas $14 \%$ possuem três ou mais doenças. $\mathrm{O}$ que chamou mais a atenção foi o fato de $90 \%$ das mulheres ingressantes do PAAF apresentarem uma ou mais doenças do sistema músculo-esquelético, enquanto apenas $21 \%$ dos homens possuem esse problema de saúde. 
LeVEILle, MANSFiELd e GURALNIK (2003) verificaram que $42,8 \%$ de sua amostra, de ambos os sexos, relataram dores músculo-esqueléticos, sendo que a artrite foi a mais apontada. De acordo os autores, as dores podem contribuir para baixar auto-eficácia para o exercício e interferir nos esforços para aumentar o nível de atividade física na população idosa. Eles afirmam que idoso com dores em um ou mais locais e com auto-eficácia diminuída possui três vezes mais chances para ser sedentários do que aquele com auto-eficácia elevada e sem dores.

Por outro lado, Leveille, MANSField e GURALniK (2003) afirmam que a participação em atividades físicas deve diminuir as dores crônicas e também ajudar a elevar a auto-eficácia, num processo que denominaram de "espiral". Melhorando a autoeficácia para o exercício, melhora a adesão e, consequentemente, melhora o controle da dor. Talvez os alunos do PAAF tenham passado por esse processo, pois em alguns relatos pessoais eles afirmaram que as dores que sentiam desapareceram, como pode ser ilustrado pelos relatos de alguns sujeitos:

(...) eu sofro da coluna, eu não sinto mais dor, meu joelho doía, precisava operar, agora não. Eu caia, batia a cabeça, agora não (ATP, 82 anos).

(...) adquirir uma segurança que eu não tinha pelos exercícios de equilíbrio, força nas pernas, flexibilidade ... melhorou muito as dores lombares (MAS);

a ginástica foi muito boa para mim, realizou muitas coisas boas, principalmente porque não senti mais dores na coluna (MCO, 62 anos).

Tais falas revelam que a participação no programa foi importante para diminuir as dores, e quiçá, tenha sido um dos fatores para sua permanência até seu final.

Com relação à atividade física pregressa dos egressos do PAAF-2002, vimos que o histórico de vida da pessoa é fundamental para a adoção de um estilo de vida fisicamente ativo na velhice. Indivíduos que foram ativos fisicamente no passado tendem a continuar ativos durante o envelhecimento. As pessoas escolherão o estilo de vida mais parecido com os padrões que elas viveram na juventude e na meia-idade (CURTIS, White \& McPherson, 2000; Langley \& Knigth, 1999). Indivíduos que foram ativos ao longo da vida possuem maior percepção sobre a eficácia de um programa e têm maior probabilidade de possuir preferências por determinadas atividades físicas. Por outro lado, os que sempre foram sedentário na juventude e na meia-idade possuem atitude negativa diante da prática de atividade física (CURTIs, WhITE \& MCPHERSON, 2000).
O que chama atenção no PAAF-2002 é que uma boa parte dos alunos iniciaram ou reiniciaram suas atividades físicas tardiamente, após os 30 anos, sendo que 10 egressos $(18,5 \%)$ iniciaram ou reiniciaram após os 50 anos. Esse mesmo índice foi a percentagem de pessoas que teve no PAAF-2002 a primeira experiência com atividade física, ou seja, começaram após os 60 anos.

Esse fato pode ser explicado à luz da Teoria da Ressocialização (ChOGahara \& Yamaguchi, 1998; Curtis, White \& McPherson, 2000). Para Chogahara e Yamaguchi (1998) esta teoria parte do pressuposto que mudanças fundamentais ou transiçōes comportamentais ou cognitivas proporcionam ao indivíduo experiências que possibilitam mudanças em suas crenças, conhecimentos e valores. Tais mudanças no estilo de vida do idoso englobam novo status social, aposentadoria, viuvez, etc.

Essas mudanças foram as conclusões que CURTIS, White e MCPHERSON (2000) chegaram para explicar o aumento no nível de atividade física de homens canadense, acima de 65 anos, no período de 10 anos. Segundo os autores, as mudanças características dessa faixa etária como aposentadoria ou o trabalho tornando-se menos central, sobrou mais tempo, energia e recursos para esses idosos dedicarem a outras atividades.E muitos priorizaram a atividade física em suas vidas.

No caso dos ingressantes do PAAF-2002, tais mudanças parecem afetar principalmente as mulheres. Como vimos nas TABELAS 8 e 9, mais mulheres apresentaram histórico de vida sedentária e também são elas que decidiram iniciar prática de atividade física mais tardiamente. Há um índice muito maior de mulheres que iniciou a atividade física após os 50 e 60 anos de idade (45\% das mulheres iniciaram nessa fase da vida, após os 50 anos, ao passo que apenas dois homens $-14,28 \%$ iniciaram após os 50 anos de idade) do que os homens. Parece que os homens são mais resistentes às mudanças no estilo de vida, como, por exemplo, adotar a prática de atividade física nas idades mais avançadas. Ou então, os homens apresentam maiores barreiras para participar de programas supervisionados de atividade física.

Vale a pena destacar o comportamento das mulheres que sempre foram donas de casa. Aqui se revelou que a maioria delas (55\%) sempre foi sedentária. Apenas uma foi fisicamente ativa desde a adolescência, uma após os 40 anos e duas após os 50 anos. A constante ocorrência desse fato nos PAAFs anteriores, nos leva a crer que pode haver forte relação entre o fato da mulher idosa ter trabalhado fora de casa e a participação em atividades física. Por isso, 
são necessárias novas investigações, que confirmem ou não essas suposições. Ao se confirmar, isso pode acarretar numa importante determinante que leva as mulheres a aderir a programas de atividade física.

Por fim, analisando os motivos de adesão inicial ao PAAF-2002, como podemos observar na TABELA 10, aprender a fazer exercícios corretamente, melhorar a condição física e funcional, prevenir problemas de saúde e aumentar o bem-estar emocional foram, na ordem, os principais objetivos a serem atingidos pelos sujeitos com sua participação no PAAF 2002. Por outro lado, auxiliar no tratamento de saúde, ocupar o tempo livre, melhorar performance esportiva, aprender a lidar com problemas de saúde e ampliar a rede de amigos, foram objetivos considerados menos importante.

A fala espontânea dos sujeitos reforça sua resposta aos objetivos inicias listados anteriormente. Analisando a frequência das respostas verificamos, na TABELA 11, que o objetivo mais citado foi o de melhorar o condicionamento físico com 30\% das respostas. Já adquirir conhecimentos (sobre exercício, sobre o corpo, sobre o envelhecimento) configurou-se o segundo item mais citado com $20 \%$ de freqüência das respostas, seguido de melhorar a saúde (19\%) e melhorar a qualidade de vida (14\%). Já as respostas que apareceram com menos frequência foram interagir socialmente, fazer amigos e ocupar o tempo livre.

Esses resultados vão, em parte, ao encontro dos achados de Hivensalo, Lampien e Rantanen (1998), que afirmam que a razão mais importante para a adesão de idosos à programas de atividade física é a preocupação com a saúde. Também, MANSFiEld, MarX e GuralniK (2003) encontraram na obtenção de melhor condição física, os principais motivos para os idosos de suas amostra participarem de programa de atividade física.

No entanto, nenhum estudo fez referências à busca de conhecimentos como um dos principais objetivos para a participação de idosos em programas de atividade física. No caso dos ingressantes do PAAF-2002, adquirir conhecimento foi o objetivo mais importante, talvez porque boa parte $(54 \%)$ dos ingressantes do PAAF-2002 procurou o programa por indicação de ex-alunos do PAAF e de alunos da EEFEUSP. Isto significa que, provavelmente, eles já sabem que no PAAF o ensino de conhecimentos teóricos tem forte relação com as atividades práticas.

Tal interpretação pode ser ilustrada pelos relatos espontâneos de alguns alunos, quando foram solicitados, ao final do PAAF-2002, a responderem sobre como descreveriam o programa para um amigo ou parente. A maioria deles (52\%) respondeu essa pergunta dando grande ênfase aos aspectos ligados aos conhecimentos aprendidos e aos aspectos pedagógicos do programa. Talvez os ingressantes do PAAF-2002 tenham recebido informações semelhantes as apresentadas:

É um curso que nos deu conhecimentos do corpo humano ou para alguns recordou com novos nomes" (AM, 62 anos).

(...) além dos ensinamentos da educação física, ministra aulas teóricas explicando os "porquês" (EE, 70 anos).

(...) É um curso que se aprende fazer exercícios físicos próprios para nossa idade (JCF, 71 anos). (...) É um aprendizado para a prática de exercícios, afim de melhorar a qualidade de vida do idoso, enfocando os aspecto que há perdas (força, flexibilidade capacidade neuromotora e aeróbias (ML, 62 anos).

(...)É um curso de atividade física (...), mas baseados em explicaçôes teóricas para que cada um venha conhecer suas deficiências e como trabalhá-las (NV, 63 anos).

(...) É um programa para o desenvolvimento das atividades físicas que irão propicia às pessoas idosas a autonomia para praticá-las regularmente, melhorando suas condições físicas, retardando ou prevenindo o aparecimento de doenças (...), aumentando o período de expectativa de vida ativa (MI, 63 anos).

Esses resultados nos levam à reflexão sobre a necessidade e a importância de programas educacionais para a população idosa. Programas que potencializem a dimensão cognitiva e que possibilitem o acesso de idosos a novos conhecimentos, por meio de processos educacionais, merecem e precisam ser desenvolvidos e difundidos, na medida em que seus egressos podem se tornar agentes multiplicadores, levando a outros os conhecimentos construídos no programa educacional, como é o caso do PAAF. Por esta razão, programas de atividade física não devem priorizar apenas aspectos físicos, funcionais e de saúde. $\mathrm{O}$ conhecimento teórico associado às atividades físicas desenvolvidas pode se configurar num importante elemento para a mudança de atitude e comportamento diante da prática de atividade física.

Vale a pena ressaltar, nos resultados encontrados, a pouca importância atribuída a objetivos como "fazer amizades" e "ocupar tempo livre", contrariando a afirmação de muitos estudiosos e profissionais. Os achados deste estudo em relação aos objetivos considerados menos importantes ou os que apareceram com menos frequência diferem completamente dos trabalhos encontrados na literatura. 
Autores como Goggio e Morrow Junior (2001) e Mansfield, Marx e Guralnik (2003), por exemplo, afirmam que as interações sociais, fazer novos amigos, ocupar o tempo livre são importantes motivos que levam o idoso a procurar programas de atividade física. A pouca importância atribuída a esses objetivos, em relação aos ingressantes do PAAF 2002, parece indicar que objetivos diretamente ligados à saúde e à melhora da qualidade de vida são muito

\section{Conclusão}

O perfil apresentado pelos ingressantes do PAAF2002 se assemelha aos de idosos de países desenvolvidos, praticantes de atividade física, principalmente, no que diz respeito às características sócio-econômicas. São idosos jovens, com alto nível de escolarização e econômico, assemelhando-se mais ao dos idosos desses países do que à maioria dos idosos brasileiros.

Eram pessoas fisicamente ativas antes de ingressar no programa, confirmando que o histórico de vida pode ser um forte determinante para prática regular de atividade física. Contudo, uma pequena parte iniciou a prática de atividades desta natureza somente após os 50 e os 60 anos de idade, talvez em decorrência das mudanças típicas da velhice, como a aposentadoria, ou viuvez.

O que chama atenção é o fato que foram as mulheres, principalmente, que iniciaram, ou reiniciaram, suas atividades físicas no inicio da velhice, o que nos leva a crer que elas têm maior capacidade para mudança de comportamento e de estilo de vida do que os homens.

Os sujeitos apresentavam grande incidência de doenças, com muitos portando mais de três tipos, chegando em alguns casos a sete doenças. Entre as mulheres, as doenças músculo-esqueléticas prevaleceram, enquanto nos homens foi a hipertensão arterial. Não obstante a alta incidência das doenças relatadas, elas não representaram uma barreira para a prática da atividade física, mas ao contrário, em alguns casos, o alívio das dores e dos desconfortos pode ter servido como incentivo para que os sujeitos continuassem a participar do programa.

O fato da maioria das mulheres que procurou o PAAF ter desenvolvido uma atividade profissional ao longo da vida, nos leva a especular que tal condição pode ser um forte determinante para a prática de mais importantes. Para muitos, ampliar os horizontes por meio da aquisição de novos conhecimentos, juntamente com a melhora das condições físicas e funcionais, é muito mais importante, e lhes garante mais significado, do que apenas poder sair de casa. Talvez esses resultados contribuam para atenuar o estereótipo de que o idoso é um "indivíduo carente" e, por isso, busca atividades em que possa estar com outras pessoas para supri-la.

atividade física entre mulheres idosas. Embora não tenhamos resultados científicos que confirmem os achados deste estudo, esse comportamento vem sendo observado a cada nova turma do PAAF.

Os motivos que levaram as pessoas procurarem $\mathrm{O}$ PAAF-2002 e os objetivos que desejavam alcançar estavam relacionados à melhora da condição física e funcional, à prevenção e melhora de problemas de saúde, à melhora do bem estar emocional e da qualidade de vida. A busca de conhecimentos sobre o corpo, sobre o envelhecimento e sobre a atividade física merece destaque, pois foi muito freqüente nas respostas dos ingressantes do programa. Por outro lado, a interação social, fazer amigos e ocupar o tempo livre foram razóes consideradas menos importantes pelos ingressantes do PAAF-2002.

Os resultados apontam também para a importância do programa educacional que prioriza aspectos práticos e teóricos. As pessoas que passaram pelo PAAF agem como agentes multiplicadores das informaçôes e dos conhecimentos aqui adquiridos. As informações sobre o programa, os conhecimentos adquiridos, a ação pedagógica, a relação professor-aluno são alguns elementos relatados por ex-alunos e que talvez tenham sido determinantes para estimular boa parte das pessoas que ingressaram no PAAF-2002.

Por fim, fica como sugestão para futuro trabalho, um estudo epidemiológico, abrangendo vários programas de atividade físicos para idosos, oferecidos por instituiçōes como clubes esportivos, academias, entidades de classe, centros comunitários, em pontos distintos da cidade. Com isso, poderíamos traçar, com maior precisão, o perfil do idoso que pratica atividade física e permitirá a elaboração de estratégias mais eficientes para atrair a atenção de idosos sedentários. 


\begin{abstract}
The profile of the initiators in a physical education program for elderly and the reasons for the initial adherence

In an aging society, Physical Education has paramount importance. Each year the search for supervised programs expends, as in the Elderly Physical Activity Autonomy Program (PAAP) case. Understanding the elderly is important to orientate the pedagogic actions and to attend participants' needs. Therefore, the purpose of this study was to determine the social demographic characteristics, previous physical activity, perceived health of the elderly and to understand the reasons for their adherence in the PAAP. Fifty four individuals ( 14 male and 40 female, $66,45 \pm 5,176$ years old) that went into PAAP took part in this study. Questionnaires were used with the following variables: type, age, education level, marital status, perceived health, previous professional activity, previous physical activity and social-economic level. To check the reasons for the first adherence, we used the Liekert table and the Liekert Internal Consistence Index. To confirm the results, we applied the Kendall Concordance Coefficient (W) and the Friedman test $\left(\mathrm{Xr}^{2}\right)$, adopting $\mathrm{p}=0.05$ as the significance level. The majority of the participants belonged to the A and B socio-economical classes and had a bachelor's degree. The majority had, at least, one disease, but the perceived health was positive. The adherence reasons reported were: learn how to perform physical exercises, to improve physical condition and to prevent health problems. Further, take up free time and to make friends were considered the less important reasons .
\end{abstract}

UnITERMs: Physical educations; Adherence; Physical activity; Elderly.

\title{
Referências
}

BARBOSA, C.R.M. Saúde, qualidade de vida e envelhecimento: a inclusão do homem idoso em programas para terceira idade. 2003. Dissertação (Mestrado) - Universidade Federal do Rio de Janeiro, Rio de Janeiro, 2003.

BOUCHARD, C.; SHEPHARD, R.J.; STEPHENS, T. (Eds.). Physical activity, fitness, and health: consensus statemente. Champaign: Human Kinetics, 1993.

CAMARANO, A.A. Mulher idosa: suporte familiar ou agente de mudança? Estudos Avançados, São Paulo, v.17, n.49, p.35-63, 2003.

CHODZKO-ZAJKO, W.J. The fall coferences examine aspects of aging and physical activity. Jornal of Aging and Physical Activity, Champaign, v.8, n.1, p.299-300, 2000.

CHOGAHARA, M.; YAMAGUCHI, Y. Resocialization and continuity of involvement in physical activity among elderly japoneses. International Review For the Sociology of Sport, Munich, v.33, n.3, p.247-89, 1998.

CURTIS, J.; WHITE, P.; McPHERSON, B. Age and phisycal activity among canadian women and men: finding from longitudinal surwey data. Jornal of Aging and Physical Activity, Champaign, v.8, n.1, p.1-19, 2000.

DISHMAN, R.K. Determinants of participation in physical activity. In: BOUCHARD, C. et al. Exercise, fitness and health. Champaign: Human Kinetics, 1988.

FRIIS, R.H.; NOMURA, W.L.; MA, C.X.; SWAN, J.H. Socioepidemiologic and health-related correlates of walking for exercise among the elderly: results from the longitudinal study of aging. Jornal of Aging and Physical Activity, Champaign, v.11, n.1, p.54-65, 2003.

GIATTI, L.; BARRETO, S.M. Trabalho feminino e saúde na terceira idade. Ciências e Saúde Coletiva, Rio de Janeiro, v.7, n.4, 2002.

GOGGIO, N.L.; MORROW JUNIOR, J.R. Physical activity, beheaviors of older adults. Jornal of Aging and Physical Activity, Champaign, v.9, n.1, p.58-66, 2001.

HIVENSALO, M.; LAMPIEN, P.; RANTANEN, T. Phisical exercise in old age: na eight-year follow-up study on evolvemente, motives and obstacles among person age 65- 84. Jornal of Aging and Physical Activity, Champaign, v.6, n.2, p.157-68, 1998. INSTITUTO BRASILEIRO DE GEOGRAFIA E ESTATÍSITICA (IBGE). Senso demográfico (2000). Disponível em: <www.ibge.gov.br>. 
KING, A.C.; MARTIN, J.E. Aderência ao exercício. In: AMERICAN COLLEGE MEDICINE OF SPORT. Prova de esforço e prescrição de exercícios. Rio de Janeiro: Revinder, 1995. p.332-41.

LANGLEY, D.J.; KNIGTH, S. Continuity in sport partcipation as na adaptive strategy in the aging process: a life span narrative. Jornal of Aging and Physical Activity, Champaign, v.7, n.1, p.32-54, 1999.

LEVEILLE, S.G.; MANSFIELD, J.C; GURALNIK, J.M. The impact of chronic musculoskeletal pain on exiercise: atittude, self-efficacy anda physical activity. Jornal of Aging and Physical Activity, Champaign, v.11, n.2, p.275-83, 2003. LITWIN, H. Social predictors of physical activity in later life: the contribution os social-network type. Jornal of Aging and Physical Activity, Champaign, v.11, n.3, p.389-407, 2003.

MANSFIELD, J.C.; MARX, M.S.; GURALNIK, J.M. Motivators and barriers to exercise on older community-Dwelling population. Jornal of Aging and Physical Activity, Champaign, v.11, n.2, p.242-53, 2003.

McKOWNA, P.; NAYLOR, N.M.; McDOWELL, N. Barriers to physical activity promotion by general practitioners ande practice nurses. Bristish Journal of Sports Medicine, London, v.32, n.3, p.242-7, 1998.

MILLS, K.M.; STEWART, A.L.; SEPSIS, P.G.; KING, A.C. Consideration of adult's preferences for format of physical activity. Jornal of Aging and Physical Activity, Champaign, v.5, n.1, p.50-8, 1997.

MOREY, M.C.; DUBBERT, P.M.; DOYLE, M.E.; MacALLER, H.; CROWLEY, G.M.; KUCHIBHATLA, M.; SCHENKNAN, M.; HORNER, R.D. From supervised to unsupervised exercise factor associated with exercise aderence. Jornal of Aging and Physical Activity, Champaign, v.11, n.3, p.351-68, 2003.

OKUMA, S.S. O significado da atividade física para o idoso: um estudo fenomenológico. 1997. Tese (Doutorado) - Instituto de Psicologia,Universidade de São Paulo,1997.

PASTORE, J. O que fazer com as viúva? Disponível em <http:// www.josepastore.com.br/artigos/ac/ac_088.htm>. Palestra realizada na Ordem dos Advogados do Brasil (OAB - Recife) em 21/03/2002.

SHEPHARD, R.J. Aging, physical activity and health. Champaign: Human Kinetics, 1997.

SHIBATA, H. An overviewof the Tókio Metropolitan Institute of Gerontology: longitudinal interdisciplinary study on aging (TMIG-LISA, 1991 - 2001). Jornal of Aging and Physical Activity, Champaign, v.8, n.4, p.35-48, 2000.

SPIRDUSO, W. Physical dimensions of aging. Champaign: Human Kinetics, 1995.

TAYLOR, C.B.; MILLER, N.H.; FLORA, J. Princípios de mudança de comportamento para com a saúde. AMERICAN COLLEGE MEDICINE OF SPORT. Prova de esforço e prescrição de exercícios. Rio de Janeiro: Revinder, 1995. p.319-25. WILCOX, S.; KING. A.C.; BRASSINGTON, G.S. Physical activity preferences of middle-aged and older adults: a community analisis. Jornal of Aging and Physical Activity, Champaign, v.7, n.4, p.386-99, 1999.

ENDEREÇO

João Raimundo Peixoto Pereira

Av. Dona Blandina Ignês Júlio, 741 - apto. 77-A o6050-120 - Osasco - SP - BRASIL e-mail: profjpereira@yahoo.com.br
Recebido para publicação: 23/07/2007

Aceito: 20/07/2009 